\title{
TWO SPECIES OF PASSIFLORA (PASSIFLORACEAE) NEW TO NEPAL
}

\author{
V. Manandhar and P. P. Kurmi
}

\begin{abstract}
The present paper includes Passiflora ciliata Ait. \& $P$. foetida L. as new records to flowering plants of Nepal. The description along with phytogeographical distribution and a key to facilitate the identification of species have been provided herewith.

Key words: Distribution, flowering plants, Nepal, Passiflora ciliata, phytogeography

\section{Introduction}

Previous publications on Nepalese flowering plants (D. Don, 1825; Hara, 1975; Bull. Dept. Med. PI. No. 7, 1976; Hara et al., 1979; and Press et al., 2000) include 3 species of Passiflora ( $P$. caerulea L., $P$. edulis Sims. and $P$. napalensis Wall.). During botanical surveys in Central Nepal, two species of Passiflora have been collected. These specimens were studied and found to be different from the previously reported species in having lacerate stipules and bracts. With the help of literatures, these species were identified as Passiflora ciliata Ait. and $P$. foetida L. (Family : Passifloraceae) and reported as new to Nepal.
\end{abstract}

\section{Materials and Methods}

The collected specimens were studied, identified and housed at National Herbarium (KATH), Godawari for future reference. Here, a key has been provided to facilitate the identification of all the species of Passiflora reported till now from Nepal. The newly reported species, indicated with asterisk marks in the key, have been provided with description and distribution.

\section{Key to the species}

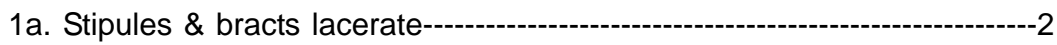

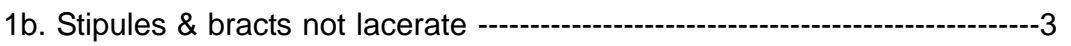

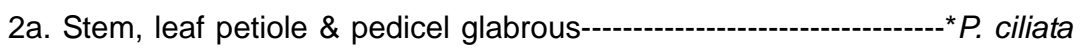

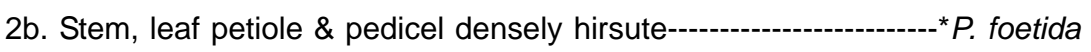

3a. Leaves unlobed, entire, stipules \& bracts linear, inflorescence, few flowered cyme $P$. napalensis

3b. Leaves lobed, entire or serrate, inflorescence one flowered-------------------4

4a. Leaves 3-lobed, serrate, stipules, linear, bracts serrate-----------------P. edulis

4b. Leaves 5-lobed, entire, stipules, reniform clasping stem, bracts entire P. caerulea 


\section{Description of the species}

Passiflora ciliata Ait., Hort. Kew, 3:310 (1789).

Herbaceous climber. Stem slightly striate, glabrous. Stipules clasping the stem, lacerate, forming an involucre of gland tipped capillaries. Leaves simple, alternate; petiole $2-4.5 \mathrm{~cm}$, glabrous, few glandular hairs present; leaf blade 3-lobed, $3.5-7 \times 2.5-7 \mathrm{~cm}$, lobes broad ovate with acute apex, base cordate, margin serrate, ciliate with gland tipped hairs, adaxially glabrous, or with very few hairs, abaxial surface with few glandular hairs towards base. Inflorescence solitary in leaf axils. Bracts 3 , lacerate or pinnatifid forming involucres of threadlike lobes with glandular tips. Flowers light purple to pink, $\mathrm{ca} 3 \mathrm{~cm}$ in diameter; pedicels 2 - 4cm, glabrous; sepals 5, $1.5-2 \mathrm{~cm}$, oblong, glabrous, green, white adaxially, apex awned; petals $5,1.5-2 \mathrm{~cm}$, pink or purple, oblong, obtuse or rounded, glabrous; corona 3 to 5 seriate, filamentous, two outer series ca. $1 \mathrm{~cm}$, inner series ca $2.5 \mathrm{~mm}$; androgynophore ca $3.5 \mathrm{~mm}$, stamens 5 , filaments 3.5 - 4mm, flat, anthers oblong; ovary ovoid to ellipsoid, ca. $5 \mathrm{~mm}$, glabrous; styles 3; stigmas capitate. Fruit $2.5-3.5 \mathrm{~cm}$ diameter, orange yellow or orange-red, ovoid or globoid, glabrous. Seeds numerous, pale brown, elliptic, $3-3.5 \mathrm{~mm}$.

Flowering : Jul. - Aug.

Fruting : Sep.. - Nov.

Ecology : 150 - 1200m, climbing over the shrubs and small trees, on open grassy places and roadsides.

Distribution : India, Nepal.

Specimens examined : C. Nepal : Tilaurakot, Kapilvastu Dist., 165m, 10. 9. 2001, P. P. Kurmi, 15998 (KATH). Jagdishpur Lake, Kapilvastu Dist., 165m, 4. 4. 2007, S. R. Baral, L. Joshi, A. P. Bhattarai \& P. P. Kurmi, 7592 (KATH).

New to Nepal.

Passiflora foetida L., Sp. PI. 2: 959. 1753.

Herbaceous climber, bad smelling. Stem striate, densely hirsute with yellowish hairs. Stipules, lacerate, deeply parted to form an involucre of gland tipped capillaries. Leaves simple, alternate; petiole $2-5.5 \mathrm{~cm}$, hirsute with occasional glandular hairs; leaf blade 3-lobed, 5 - 10 X $4-8 \mathrm{~cm}$, lobes broad ovate to oblong-ovate with acute apex, base cordate, margin undulate, ciliate, marginal hairs mixed with glandular hairs, adaxially sparingly hairy mixed with glandular hairs, abaxial surface with few hairs along veins. Inflorescence solitary in leaf axils. Bracts 3 , lacerate or pinnatifid forming threadlike lobes with glandular tips. Flowers light purple with white tinges, $2-4.5 \mathrm{~cm}$ in diameter; pedicels $1.5-4 \mathrm{~cm}$, hirsute; sepals $5,1.5-2 \mathrm{~cm}$, oblong, ciliate, pale green, white adaxially, apex awned; petals $5,1.5-2 \mathrm{~cm}$, white, oblong, obtuse; corona 3 to 5 -seriate, filamentous, two outer series ca. $1 \mathrm{~cm}$, inner series ca $2.5 \mathrm{~mm}$; androgynophore $5-6 \mathrm{~mm}$, stamens 5 , filaments $4-5 \mathrm{~mm}$, flat, anthers oblong; ovary ovoid to globoid, ca. $5 \mathrm{~mm}$, glabrous; styles 3 ; stigmas capitate. Fruit $2.5-3.5 \mathrm{~cm}$ diameter, orange yellow or orange-red, ovoid or globoid, glabrous. Seeds numerous, pale brown, elliptic, 3 $3.5 \mathrm{~mm}$. 
Flowering : Jul. - Aug.

Fruiting : Aug. - Nov.

Ecology : 150 - 1200m. Climbing over the shrubs and small trees of roadsides, forests and riverine sandy places.

Distribution: India, China, Nepal.

Specimens examined : C. Nepal : Butawal, Rupandehi Dist., 200m, 15. 11. 2005, P. P. Kurmi, 7486 (KATH). Tinau Khola, Butawal, Rupandehi Dist., 200m, 18. 12. 2005, M. K. Adhikari, L. Joshi, V. Manandhar \& P. P. Kurmi, 13 (KATH).

New to Nepal.

\section{Acknowledgements}

The authors are grateful to Dr. Lokendra R. Sharma, Director General, Dept. of Plant Resources and Dr. S. R. Baral, Chief, National Herbarium \& Plant Laboratories, Godawari, for providing necessary facilities. Dr. Mark F. Watson, Royal Botanic Garden, Edinburgh and Ms. Sajan Dahal, NHPL, Godawari, are also thanked for their moral support to prepare this manuscript.

\section{References}

Annonymous. (1976). Bull. Dept. Med. Pl. (7):90.

Don, D. (1825). Passiflorae . Prod. Fl. Nep.:63.

Hara, H. (1975). Passiflorae. F. of E. Himal..Ed. (3):85.

Hara, H., Chater, A. O. \& Williams, L. H. J. (1979). An enumeration of the flowering plants of Nepal. Vol. 2. British Museum (Natural History), London:177.

Press, J. R., Shrestha, K. K. \& Sutton, D. A. (2000). Annonated checklist of the flowering plants of Nepal. Natural History Museum, London:232-233

\section{Author's Address}

${ }^{1}$ Vidya Manandhar and ${ }^{2}$ P.P. Kurmi National Herbarium \& Plant Laboratories

Godawari, Lalitpur, Nepal. 